\title{
LAS MANOS: EL LENGUAJE ESTÉTICO- TEOLÓGICO DE LA SOLIDARIDAD. INTUICIONES PARA UNA TEOLOGÍA SIMBÓLICA ${ }^{1}$
}

\author{
OLGA CONSUELO VÉLEZ CARO² \\ SILVIA SUSANA BECERRA MELO ${ }^{3}$ \\ ÁNGELA MARÍA SIERRA GONZÁLEZ ${ }^{4}$
}

Fecha de recepción: agosto 2018

Fecha de aceptación y versión definitiva: julio 2019

\begin{abstract}
RESUMEN: Tres investigaciones en pastoral urbana con población en situación de desplazamiento forzado en Ciudad Bolivar, Localidad de Bogotá, nos ha dado la pauta para adquirir nuevas comprensiones sobre el quehacer teológico. En este artículo se entrelaza la teología con el mundo simbólico que representa la corporeidad de mujeres cuyos cuerpos se convierten en relatos vivos que, con la ayuda de la estética, se transforman en textos de lectura para abrir caminos de re-significación teológica y de salvación integral.
\end{abstract}

PaLABRAS CLAVE: manos; corporeidad; estética; teología simbólica; re-significación.

\section{Hands: The theological-aesthetic language of solidarity. Intuitions for a Symbolic Theology}

ABSTRACT: Three urban ministry research projects were carried out with displaced populations in Ciudad Bolivar (Bogotá, Colombia). These initiatives have allowed us to gather new insights and understanding about the imperative theological endeavour. This article intertwines theology with the symbolic world that represents the physicality of women whose bodies have become living stories and who, with the help of aesthetics, have become reading texts which opens possibilities to theological re-signification and integral salvation.

KEY WORDS: hands; corporeity; aesthetic; symbolic theology; resignification.

1 Este es un artículo de reflexión, producto de la investigación denominada $\mathrm{Li}$ neamientos teológico-pastorales para el acompañamiento a personas en situaciones de desplazamiento. Un aporte de la pastoral sub urbana (2014).

2 Profesora titular de la facultad de Teología en la Pontificia Universidad Javeriana (Bogotá, Colombia). Correo electrónico: ocvelez@javeriana.edu.co

3 Profesora asistente del Centro de Formación Teológica de la Facultad de Teología Universidad Javeriana (Bogotá, Colombia). Correo electrónico: sbecerra@javeriana.edu.co

4 Docente de la facultad de Teología de la Universidad Javeriana (Bogotá, Colombia). Correo eletrónico: angela.sierra@javeriana.edu.co 


\section{INTRODUCCIÓN}

A lo largo de tres investigaciones con el grupo de Teología y Género, siendo estas, "Curar y levantar los cuerpos femeninos una lectura desde la hermenéutica crítica feminista ${ }^{5}$, «El desplazamiento forzado un desafío a la pastoral urbana $»^{6} \mathrm{y}$ «Lineamientos teológico pastorales para el acompañamiento a personas en situación de desplazamiento. Un aporte desde la pastoral (sub)urbana $»^{7}$ nos ha permitido constatar la centralidad del cuerpo como categoría hermenéutica ${ }^{8}$. Comenzamos a descubrir que la corporeidad de las mujeres que asistían a nuestros talleres en Ciudad Bolívar se convertía en el primer texto para recuperar la memoria y el sentido. Y así, siguiendo la intuición de la primera investigación continuamos indagando la riqueza de lo corporal como símbolo de liberación y como camino para nuevas construcciones?.

5 Vélez y Sierra, Investigación financiada por la Vicerrectoría Académica de la Pontificia Universidad Javeriana (2009-2010), ID PRY 003703.

6 Esta investigación se llevó a cabo bajo el título El desplazamiento forzado: un desafío para la pastoral (sub)urbana (2011-2013). Los resultados de la misma pueden consultarse en Vélez, Sierra, Becerra, Rodríguez (OAR) \& Rozo.

7 Esta investigación está apoyada por la Pontificia Universidad Javeriana, (marzo 2014-septiembre 2015), ID PRY 006010.

8 Ya la teología feminista había evidenciado esta categoría en los estudios bíblicos y en la totalidad de la teología. El cuerpo para las mujeres comenzó a ser el primer texto para interpretar y entender la relación personal con Dios y con los otros. El texto es un cuerpo que se muestra y se oculta a sus lectoras/es. Aquellos y aquellas que leen también son cuerpos vivientes que entran en diálogo y lucha con otro cuerpo: el texto. Ambos muestran sus propios tejidos: cuerpos individuales y sociales, femeninos y masculinos. Entender el texto como cuerpo, fruto de relaciones sociales de género, entender el proceso de interpretación también a partir de las relaciones concretas de los cuerpos, trae nuevas luces para la comprensión del discurso. El cuerpo como criterio hermenéutico ofrece alternativas de lecturas nuevas que invitan a vivir nuevas relaciones entre mujeres y hombres: en la teología, en las iglesias, en la casa, en la cama y en la vida (Cardoso, 2015).

9 En el primer taller se trabajó con 6 varones y 9 mujeres (personas en situación de desplazamiento); 4 agentes de pastoral y 5 organizaciones que trabajan con desplazados. En esa ocasión se utilizaron las técnicas de entrevista a profundidad, grupo focal y un taller con el objetivo de sistematizar la manera como las iglesias están respondiendo a las personas desplazadas y, al mismo tiempo, las expectativas que estas personas tienen frente a las iglesias. Una descripción más completa de este trabajo de campo se puede consultar en Vélez et al. (2014, pp. 222-224, pp. 230-232). En el segundo taller se trabajó con 10 mujeres, en un tema más enfocado al símbolo de las manos como posibilidad de leer su historia motivación primera para elaborar este artículo por la riqueza que surgió en todas las dinámicas. 
La corporeidad ${ }^{10}$ de la población desplazada, en cada encuentro que realizamos, evidenciaba otros asuntos que debíamos atender de manera urgente: cicatrices, duelos y estos a través de la lectura del símbolo profundo que representaban ciertas partes del cuerpo como los pies, las manos, el pecho, la espalda. "Hay una conexión profunda entre la memoria y la realidad física del cuerpo. Están muy unidos el cuerpo y la memoria en las personas desplazadas por eso sufren muchas enfermedades físicas, sicológicas y espirituales»(Rodríguez y Sierra, 2013, p. 276).

De manera paralela se iba evidenciando una experiencia de fe muy conectada con la experiencia corporal, como eje central para soportar y superar la crisis del desplazamiento, en imaginarios tanto negativos como positivos. Con expectativas y esperanzas nuevas.

Esta primera indagación y lo que, posteriormente, fue una constatación nos impulsó a seguir ahondando en la importancia de lo corporal, pero, sobre todo, en el camino que se abre para la teología desde este tipo de experiencias.

Las intuiciones que emergieron en los talleres con las mujeres, realizados en noviembre de 2015, nos motivaron para realizar una búsqueda bibliográfica que nos permitiera fundamentar la lectura teológica de lo corporal. Es así como surge la hipótesis de trabajo que se desarrollará en este artículo.

La corporeidad ${ }^{11}$ en el sujeto víctima del desplazamiento se transforma a través de la contención de la comunidad en espacio existencial de sentido, gracias a la dimensión estética teológica, donde el símbolo de las manos y la centralidad del tocar se convierte en camino para lograrlo.

La incorporación a una verdadera comunidad y el sentirse pertenecientes a ella es una nostalgia y búsqueda constante en este tipo de población, pero es algo que se logra procesualmente.

Muchas de estas mujeres llegaron al espacio de Vidas Móviles ${ }^{12}$ desintegradas por todo lo que habían vivido durante el desplazamiento. Sus enferme-

10 La investigación también nos fue indicando la importancia del tránsito semántico del cuerpo a corporeidad que se explicará más adelante.

11 «La corporeidad es la forma visible en y mediante la cual el ser humano se hace presente al otro y se comunica; esta presencia no es sólo un dato de orden espacial, sino de orden personal, es decir, requiere el reconocimiento de sí como yo personal; así el cuerpo se establece como epifanía de la persona: es lenguaje y comunicación capaz de expresar acogida y don, invocación y afirmación de sí. Pero, aunque el rostro condensa en sí el sentido de esta epifanía, no hemos de olvidar que todo el cuerpo tiene una significación social, de intercambio y de encuentro, ejemplo de ello puede ser el baile y el lenguaje táctil» (Roccetta, 1994, p. 14).

12 Programa de responsabilidad social de la Pontificia Universidad Javeriana, en la localidad de Ciudad Bolívar, Bogotá. 
dades representaban un cúmulo de problemáticas en todas las dimensiones de la vida. Sin embargo, al escucharlas después de una participación activa en los grupos y en los talleres reconocemos que ha sido fundamental el entramado de personas que en su momento las acogieron y las promovieron. Hay unas características especiales en estas dinámicas comunitarias que les permitieron recuperar su dignidad. Por eso queremos evidenciar estos aspectos sutiles pero profundos en esta nueva fase de la investigación los cuales nos permitirán comprender el paso del cuerpo a la corporeidad y en esta dinámica potenciar el símbolo de las manos y del tocar desde la perspectiva teológica ${ }^{13}$.

Las violencias hacia las mujeres no son un fenómeno nuevo, aunque si lo sean su reconocimiento como una problemática social, con efectos sobre el cuerpo social e implicaciones en el cuerpo político, que demandan otro tipo de análisis y de acciones. A lo largo de los últimos años, las denuncias de las mujeres y de las activistas femeninas han obligado a la academia, a los diversos organismos internacionales y a los Estados a estudiarlas y considerar estrategias para su visibilización y la búsqueda de soluciones. La decisión más destacada ha sido la de conceptualizar esta realidad, lo cual, como lo ha señalado Celia Amorós, equivale a politizar su existencia.

(Gutiérrez, 2012, p. 15)

\section{LA CORPOREIDAD: UNA SENDA DE SIGNIFICACIÓN}

Procesualmente en la investigación fuimos descubriendo la riqueza y aporte de la corporeidad de estas mujeres de una manera integral. Pero fue el ámbito estético el que nos invitó a comprender la centralidad de lo corporal y sus beneficios, a dicha dimensión le dedicaremos un apartado especial para ampliar la comprensión de sus beneficios y su relación estrecha con la teología. Por ahora es importante comprender que en la historia de estas mujeres se ha dado un paso del cuerpo-individual a la pertenencia de un cuerpo-colectivo a través de la experiencia de dos movimientos vitales: por

13 En los primeros talleres trabajamos con hombres y con mujeres, pero en la segunda fase de la investigación el grupo se redujo únicamente a mujeres por esta razón: «En la investigación que realizó el grupo Teología y Mundo Contemporáneo de la Pontificia Universidad Javeriana sobre 'Pastoral urbana y desplazamiento', se pudo constatar que las mujeres se sitúan con más facilidad que los varones en las grandes ciudades, porque logran trabajar en el rol doméstico que siempre han desempeñado. Ahora bien, toda esta realidad no está exenta de muchos desafíos» (Vélez, 2014, p. 460). 
un lado, la posibilidad de sentirse contenidas y por otro transformarse en hacedoras de comunidad. Para evidenciar estos aspectos vamos a recurrir a la lectura paralela de escenas puntuales en los textos bíblicos donde se hace evidente el sentido del tacto como símbolo de salvación personal, pero al mismo tiempo de apertura a lo comunitario y la narrativa de estas mujeres las cuales hacen procesos similares.

De esta manera, tejeremos a través de los relatos bíblicos y de los relatos de nuestras mujeres la posibilidad de descubrir en sus propios cuerpos, y en con-tacto con otros/as, un nuevo significado y sentido de su realidad como corporeidad, como encuentro con otros que me construyen, superando así un sentido individual de lo corporal lo cual contradice la dignidad y salvación que ofrecen los encuentros en el evangelio y que resultan sanadores.

Esta dinámica abre la posibilidad de re-leerse y re-encontrase a través de un otro comunitario que les dignifica a través del reconocimiento, no sólo verbal sino también táctil, abriendo paso a la corporeidad. Así a través de la escucha activa ${ }^{14}$ de los duelos tatuados en la piel, de la narrativa de los acontecimientos vividos, de las emociones sentidas y compartidas, no sólo se emprende un camino sanador de regreso a sí mismas sino que también se posibilita la construcción de un relato colectivo de recuperación y tejido humano. Descubriendo en estos movimientos una visión integradora del cuerpo en la dialéctica de la corporeidad.

La identidad se basa antes de todo, en el cuerpo físico, que lejos de ser un dato «bruto», un mero acontecimiento biológico y fisiológico, es siempre objeto de interpretación y en este aspecto puede decirse que es construido. La sociedad le atribuye espacios y lugares diferentes, que condicionan y conforman su identidad y su existencia... por lo que se ha dicho hasta ahora, se hace evidente que existe una relación entre el cuerpo físico y el cuerpo social.

La antropóloga Mary Douglas así lo estudia: el cuerpo físico es un microcosmos del cuerpo social. Las actitudes de la sociedad en relación al cuerpo personal reflejan las actitudes que la sociedad tiene en relación a sí misma. El cuerpo humano lleva consigo una interpretación socio-cultural que le confiere significados y valores diferentes.

(Bernabé, 2014, p. X)

14 Realmente de lo que se trata aquí es de una Escucha compasiva la cual implica no sólo oír sino compenetrarse con la situación del otro. Está comprobado que la persona siente que se le escucha más cuando el oyente ve y se apropia incluso de las posturas del escuchado. Así al reflejar los movimientos del otro, en términos de contención, garantiza el éxito de la escucha. 
La investigación ha constatado este salto cualitativo del texto personal al texto colectivo. Estas mujeres deben recuperar su identidad desde su única posesión que son ellas mismas en sus cuerpos, pero en medio de una dinámica social contenedora que le proporcione reconocimiento y valía.

La Identidad es la parte del auto-concepto de un individuo que deriva del conocimiento de su pertenencia a un grupo social, asociado al significado valorativo y emocional surgido de esta pertenencia. Los seres humanos se comprenden a sí mismos a través de una serie de características provenientes de la pertenencia a un grupo.

(Bernabé, 2014, p. X)

«Soy porque somos», afirma el proverbio africano. En la concepción de cuerpo $^{15}$ se entrelazan aspectos sociales, de género, matices étnicos e incluso tintes religiosos, los cuales configuran una actitud frente a la corporalidad. ${ }^{16}$ La siguiente cita puede ejemplificarlo:

Con respecto a la religión, las eco feministas señalan que las tradiciones patriarcales dominantes de la civilización occidental se basan en una espiritualidad que busca, en forma bastante desesperada, trascender la naturaleza y el cuerpo - especialmente el femenino-. En contraste, este mismo grupo que se interesa en la dimensión espiritual de la vida, durante años ha tendido hacia orientaciones y prácticas que consideran a la naturaleza y cuerpo como sagrado y fuente de revelación espiritual.

(Citado en Res, 1994, p. 481)

Otro ejemplo podría ser la concepción de cuerpo que aparece en las Escrituras:

El hombre no tiene un cuerpo, una parte material, sino que es un cuerpo. No es un ser que posee un instrumento material que usa para algunas actividades, en tanto que su auténtico "yo» está identificado con su ser espiritual. No se da en la biblia una división natural del hombre, la concepción del hombre como un compuesto de alma y cuerpo no es bíblica, sino que procede de la filosofía griega.

(Pastor, 1995, p. 53)

15 Este último apartado del numeral 1, integra la investigación Vivir en la Ciudad Hoy, realizada en México (2013), que reunió las experiencias de varios países latinoamericanos en torno al tema de la Pastoral Urbana (Rodríguez y Sierra, 2013, pp. 264-265).

16 Para una mejor comprensión del texto es importante aclarar que el término corporalidad estará referido a la conciencia corporal que suscita la relación con otros; este proceso de madurez y comunicación permite construirse como persona y apertura la posibilidad de tejer comunidad. 
Se hace necesario, entonces, recuperar la noción de cuerpo del encierro de la racionalidad. Hasta hace poco tiempo el cuerpo se consideraba como tabú; a partir del movimiento existencialista, la dimensión humana adquiere un nuevo relieve dentro de la reflexión filosófica. Estamos en la «era del cuerpo» donde se re-descubre la corporeidad, su significación personal y se entiende como palabra (Porcile, 1995, p. 127).

«El modo de vivir el propio cuerpo, la relación con él se convierte en un símbolo del mundo contemporáneo occidental» (Porcile, 1995, p. 127); lo anterior invita a retomar la reflexión sobre la corporeidad, tal como la entiende la actual antropología teológica cristiana. Esta nueva visión antropológica «puede convertirse en núcleo de transformaciones necesarias para la construcción de una comunidad humana»(Porcile, 1995, p. 128).

\subsection{El CAMino de la estética, más aLLÁ de UNA HERRAMIENTA}

«La palabra estética proviene del griego aisthetikê (aisthesis: 'sensación', 'sensibilidad'), es decir que abarca todo el amplio campo de lo que es sensible al ser humano o a las percepciones sensoriales que lo conducen a una experiencia humana. Es "percibir por los sentidos», acción que en el medioevo se asoció con la palabra conocimiento para referirse a un tipo de conocimiento sensorial» (Peña, 2015).

Para Zubiri la reflexión estética no se enfatizará sobre la belleza de la obra de arte sino sobre el sentimiento estético, ${ }^{17}$. «Rescatar el sentimiento estético en un mundo como el de hoy en el que reina la confusión, el aturdimiento y el estado de angustia sería una buena "cura de urgencia» para esa anunciada regeneración espiritual del siguiente siglo».

(Borrego, 2015, p. X)

17 Otro efecto de la belleza es la capacidad de transformación personal ¿De dónde procede entonces este poder transformador de lo estético y de la música, o de lo bello en general? De la capacidad que tienen para transportarnos de un nivel de existencia a otro. Lo estético, como la música, la pintura o el buen cine (aunque sin igualarse a ellos), produce experiencia, experiencia sensorial, nos introduce por inmersión en una realidad expresiva abierta que es portadora de verdad «La verdad es el alma de la belleza. Quien no sepa acercarse a la verdad y gustar sus delicias, prostituye el concepto de la belleza, que existe y tiene su vigencia en el imperio de lo real, convirtiendo lo que es gozoso y a la vez profundo juego en el más fútil de los pasatiempos» (Guardini, op. cit., p. 80). Del Valle Caraballo, «Hasta que vuelva...» (1 Cor 11, 26). Belleza y liturgia (2012, pp. 131-143). 
Siguiendo esta línea antropológica y social, a propósito del tema, podemos decir que «toda experiencia estética constituye un triunfo de la vida humana, un momento privilegiado en el que llega el hombre a lo mejor de sí mismo», pues la relación humana no está hecha en «esquemas lineales», fríos o sin sabor, sino en "esquemas de doble dirección (apelación-respuesta; ofrecer-acoger activamente» (López Quintas, 1991, p. 8) cargados de expresión afectiva, racional y emotiva. Esta expresión lleva al ser humano a sentirse íntegro, por la aceptación y respuesta que le afirma el ser en su valor y autoestima, ofreciéndole gran apertura para actuar y medios innumerables de expresión a través de sus sentidos; «forma de integración que florece en perfección ética, en equilibrio espiritual y en eclosión de belleza» (López Quintas, 1991, p. 8) que no es otra cosa que vivir en la alegría de Cristo (Jn 17,23) (Borrego, 2015, p. X).

La importancia del término corporeidad comenzó a situarse y a empoderarse en la urdimbre de la investigación, gracias a la lectura estética de cada historia de vida; definitivamente fue nuestro contacto con la población desplazada, en rostros concretos, lo que proporcionó una pista para dar continuidad a la lectura teológica de esta experiencia, esto teniendo presente que la teología y la estética tienen como denominador común transformar, crear, encontrar sentido y significado. Algunas de estas mujeres fueron elaborando obras de arte con material reciclado y al mismo tiempo iban transformándose desde dentro. Esta es precisamente la acción estética un reconocimiento de lo bello en medio del dolor.

La narración del relato en medio del encuentro y de la escucha nos llevó más allá del discurso. Por eso tenemos que indagar otro tipo de comprensiones, cuyas respuestas nos remiten ciertamente a la profundidad simbólica de lenguaje estético-teológico.

Mirar, oler, tocar, degustar pertenecen a la narrativa que necesita una perspectiva más desde lo sensitivo, que desde lo argumentativo. Hecho que justifica el apartado siguiente.

\subsection{El CAMiNO ESTÉtico a travÉS DE los SENTIDOS. El tOCAR y El SIGNIFICA-} DO DE LAS MANOS

El tocar, en y desde su finitud, está precisamente abierto ya a una presencia sin imagen y sin representación, a una proximidad íntima que jamás se vuelve posesión, a una exposición desnuda a lo inaprehensible. El exceso sobre mí de lo que toco y de lo que me toca se verifica en la caricia.

(Derrida, 2011, p. 370) 
Las relaciones llegan a su culmen cuando hay posibilidad de encuentro a través del contacto. Las personas se transforman construyendo relaciones a través del tocar.

Lo opuesto al agarre es la caricia, pues es imposible acariciar por la fuerza, ya que la experiencia se convertiría al momento en un maltrato. Para acariciar debemos contar con el otro, con la disposición de su cuerpo, con sus reacciones y deseos. La caricia es una mano revestida de paciencia que toca sin herir y suelta para permitir la movilidad del ser con quien entramos en contacto. Mano acompasada que intenta reproducir en sus movimientos la dinámica caprichosa de la vida. Mano que renuncia a la posesión y que aprende del otro en un suave coqueteo. La ambigüedad del actuar humano pendula siempre entre la antipatía de la alergia y la posibilidad de adentrarnos en la piel del prójimo que nos brinda la caricia. La caricia, como dice Jean Paul Sartre, no es un simple roce de epidermis: es, en el mejor de los sentidos, creación compartida, producción, hechura.

(Restrepo, 1994, p. 53)

No podemos vivir sin algún tipo de caricia o roce humano. «El cuidado se teje en la escucha empática, la proximidad afectiva, el diálogo interactivo más corporal que verbal: hablan los ojos y las manos acariciando». En este sentido como afirma Francesc Torralba, urge recuperar la caricia como lenguaje de la ternura, educando en ella como expresión humana hay momentos en la vida en el que el único lenguaje del amor es la caricia. En los extremos de la vida, en tránsitos como el nacimiento y la vejez lo que mantiene conexión y comunicación humana es la posibilidad de tocarse. Tanto para integrarnos a la vida como para despedirnos de ella...necesitamos la afirmación del contacto concreto, intencional y empático.

La expresión por excelencia de la ternura es la caricia, la caricia es un tipo de contacto donde se pone de relieve la proximidad ética y el respeto al otro...la caricia es una forma excepcional para expresar la cercanía y el cuidado solícito.

(Martínez, 2012, p. 156)

Las caricias ayudan a la salud física, psicológica y emocional. Y cuando no la usamos nos privamos además de disfrutar de una placentera inundación de endorfinas: sustancias hormonales que produce nuestro propio organismo en respuesta a la estimulación táctil y las cuales tienen un efecto analgésico y producen una gran sensación de bienestar.

(Citado en Lizcano, 2014, p. 113)

Al tocar, percibo cuales son las cualidades de lo tocado. Lo que toco produce un sentimiento en mí, una posibilidad de transformación...pone algo en movimiento en mí, mueve mis emociones y me llega al corazón...hay una conmoción interior (Grün, 2010, p. 90). 
El mismo Jesús se deja tocar en un momento de gran vulnerabilidad: en una situación de angustia y de temor, recibe el contacto, la proximidad y la caricia de una mujer que lo unge con perfume (Jn 12,3). Cuentan que, poco antes del prendimiento, Juan estaba recostado sobre el pecho de Jesús (Jn 13,23): es una escena de mucha cercanía física ¿Estaría orando por él? ¿Compartiendo un momento de intimidad con el amigo en tiempos difíciles cuando ya no llegan las palabras? ¿No es ese a veces el único gesto que necesitamos hacer: dejarnos reposar y descansar y sabernos inmerecidamente acogidos en ese espacio mayor que nos sostiene?

Etty Hillesum lo expresa hermosamente: ...En un momento de descuido y de abandono, me encuentro de repente en el pecho desnudo de la vida. Sus brazos me rodean muy suavemente, me protegen, y soy casi totalmente incapaz de describir los latidos de su corazón: son tan lentos y regulares y suaves..., casi apagados, pero constantes, como si no quisieran parar jamás. Son también muy buenos y piadosos.

(Citado en López Villanueva, 2010, pp. 409-414)

\section{EL SÍMBOLO DE LAS MANOS EN LAS MUJERES DE CIUDAD BOLÍVAR}

\section{1. ¿POR QUÉ LA MANO?}

Pero... ¿cómo surge lo propiamente humano?, y con qué se asocia este crecimiento del cerebro. Se ha dicho frecuentemente que la historia de la transformación del cerebro humano tiene que ver con el uso de instrumentos, principalmente con el desarrollo de la mano en su fabricación.

No comparto esta opinión pues la mano ya estaba desarrollada en estos antecesores nuestros. Me parece más factible que la destreza y sensibilidad manual que nos caracteriza haya surgido en el arte de pelar las pequeñas semillas de las gramíneas de la sabana, y en la participación de la mano en la caricia por su capacidad de amoldarse a cualquier superficie del cuerpo de manera suave y sensual.

(Maturana, 2015).

Si hay un símbolo por excelencia de la contención es la mano. Esta posibilita la vinculación inmediata entre seres humanos y da apertura a la comunicación, por eso hemos centrado nuestro interés en la mano como un símbolo y en el tacto como un significado ${ }^{18}$.

18 De hecho, uno de los talleres fue el de con-sentir las emociones, a través de este las mujeres iniciaron con un momento de consentir su propio cuerpo para dar paso al consentir las emociones. Surgen emociones como el miedo, la rabia, la tristeza, 
Al igual que el lenguaje o la música, la mano nos habla. Junto con la palabra, la mano es uno de los medios más expresivos con que cuenta el lenguaje humano. Es el órgano por excelencia para entrar en contacto con los otros. La mano se tiende hacia el cuerpo del otro, tratando de conjugar la distancia y abolir la separación, adaptándose a sus formas para la caricia o el masaje. La mano es el miembro del cuerpo que con más frecuencia se menciona en la Biblia: más de 1500 veces, traducido por la palabra yad, que es la letra yod del tetagrama YHWH (El Señor). En la tradición Judeo-cristiana, la mano representa el conocimiento, el poder y la autoridad. Puede servir para conocer y para amar.

(Aubin, 2013, p. 104).

El trabajo realizado en Ciudad Bolívar, nos mostró una clave antropológica que exigió buscar en la Teología una nueva categoría de lectura —en este aspecto vale la pena indagar también por su dimensión estética- . Se abre paso a la investigación en el mundo de los sentidos y, en este caso, al significado de: la mano y el tocar.

Es importante recuperar de los talleres realizados con las mujeres todo lo que lograron expresar acerca de las manos. Plasmamos algunos testimonios y nos detendremos en puntos transversales para su interpretación. Así como el significado que los artistas, los que acompañaron los procesos, dieron también a sus manos.

«Mis manos son... », dice cada una de ellas, dando sentido a su trabajo y a su ser; recrean las palabras de Clarisa Pinkola:

El acceso directo, el camino más fácil, siempre se desmorona. Entonces uno vuelve a la vida hecha a mano. Uno tiene que recoger dolorosamente las piezas de nuevo juntas, manteniendo el patrón general en la mente de uno, pero trabajando con paciencia, pieza por pieza.

(Citado en Toko-pa, 2016)

«Incomparables, inconfundibles, únicas, con mis manos oro a Dios, saludo, plasmo dibujos, palpo cosas, me visto, me arreglo, comparto cosas importantes con mis compañeros». (Participante $1^{\circ}$ ).

Nuestras manos son el espejo de nuestro cuerpo, nos muestran lo que podemos hacer con ellas, desencadenándolas del sufrimiento que puedan tener. Derramando en ellas no sangre sino lluvia de bendiciones que, con nuestras oraciones, Dios ilumina con su poder de sanación.

Si nosotros creemos en el don de Dios nunca tendremos sed de su palabra y siempre beberemos agua del manantial para no caer en el pozo profundo que en

pero también en el compartir colectivo, las participantes se dan cuenta de la transformación de estas emociones en términos de fuerza, alegría y agradecimiento. 
este caso podrían ser las adversidades y sufrimientos, pero si andamos de la mano de Dios podemos salir de allí.

(Participante $2^{\circ}$ )

«Señor te doy gracias por darnos unas manos para trabajar y para luchar»

(Participante $3^{\circ}$ )

«Nuestras manos expresan, amor, comprensión. Con ellas acariciamos, pero también maltratamos. Con ellas creamos imágenes, objetos. Nuestras manos reflejan el pasar de los años y el sufrimiento que hemos vivido».

(Participante $\left.4^{\circ}\right)$

Mis manos, una obra hecha por Dios que tanto le agradezco por ese don tan privilegiado que me ha dado. Mis manos son suaves a pesar del trabajo que me ha tocado desempeñar en la vida. Me duelen debido a la responsabilidad en el hogar, pero a pesar de todo le entrego todos los días a Dios ese sacrificio y con ellas acaricio y brindo cariño, suavizo el dolor. De pronto golpeo o cuando me da malgenio pero después pido perdón.

$\left(\right.$ Participante $\left.1^{\circ}\right)$

\author{
Mis manos claman dolores \\ Acarician y construyen \\ Mis manos son creativas \\ Pero ellas jamás se destruyen \\ Con ellas alabo a Dios \\ Y las junto para orar \\ Con ellas escribo versos \\ Que esta imagen me hizo inspirar.
}

(Participante $\left.2^{\circ}\right)$

Señor: Tú nos hiciste a tu imagen y semejanza, tú me diste mi cuerpo. Pero mis manos me las regalaste para descubrir mi cuerpo, para consentirlo, para cuidarlo ya que otras manos lo han maltratado. Son para trabajar, pero también para acariciar, cuidar, proteger, defender. Para querer y acariciar mis manos son el centro de mi vida, sin mis manos no hubiera podido descubrir mi cuerpo, mi sensibilidad, mi fortaleza, mi creatividad. También el dolor cuando por una razón otras manos nos señalan, quiero que mis manos traigan a mi vida alegría para mí y para los míos. Pera defenderme y protegerme, consentirme y descubrirme. Señor, con mis manos oro y te pido que me las bendigas siempre, para dar mis manos a mí y a los demás.

$\left(\right.$ Participante $\left.5^{\circ}\right)$ 
Con nuestras manos damos muchas cosas amor caricias, con nuestras manos damos cosas a los que están a nuestro lado damos caricias a nuestra madre, a nuestros hijos, a mi esposo y a mis nietos y con nuestras manos damos alabanzas a Dios, nuestro creador. Con nuestras manos sembramos plantas y alimentos, construimos nuestras metas que nos proponemos hacer para que nazcan frutos buenos, si todo lo hacemos con fe y amor.

(Participante $\left.6^{\circ}\right)$

Mis manos donadoras de vida y a la vez reciben vida. Saben dar amor, fortaleza, esperanza y ánimo. En esta medida. Tener la capacidad de recibir, todo lo bueno que me brindan. Sin egoísmo ni hipocresía. Es muy importante hacer de nuestras manos, unas manos puras sanas que dejen huellas por cada sitio en el que en el que nos encontremos. Huellas de una caricia, de agradecimiento Nuestros manos son únicas con ellas creamos acariciamos expresamos. Ellas suelen expresar más que mil palabras.

Nuestras manos suelen ser frágiles, manos que sufren. Cuando nacemos expresan amor expresan sentimientos únicos y verdaderos. Hagamos que esto no cambie. Siempre utilicémosla para algo útil e interesante. No ensuciemos nuestras manos tomando lo ajeno, ni tampoco dejando las marcas en la piel de otro con nuestros golpes. Hagamos de ellas algo interesante.

(Participante $\left.7^{\circ}\right)$

La voz de los artistas también otorga significado a las manos:

Mis manos son mi principal órgano ya que con ellas puedo dar forma a toda mi inspiración, permitiéndome expresar todos mis sentimientos, ayudándome a comunicar mis estados de ánimo. Es mi gran comunicador después del habla, si no pudiese hablar yo sé que me comunicaría con las manos. Aristóteles dijo: 'La mano es el instrumento de instrumentos', y tenía toda la razón, yo puedo agarrar, empujar, soportar, sujetar, acariciar, pero sobre todo en mi caso expresar todo lo que $\operatorname{soy}^{19}$.

\section{Otro artista expresa:}

Pues son las manos el mayor milagro

Que de la obra de la creación el cielo hiciera

Aunque muchos no las valoran, iquién creyera!

Que las levanten, observen, y digan lo contrario.

De la mano del creador salieron

A imagen y semejanza de su creadora mano

Su obra en nuestras manos, para que, con nuestras manos,

El mundo más humano hiciéramos.

19 Así lo expresa Ángela Rivas, una profesora de artes plásticas del programa Manos de Esperanza, dirigido a las mujeres, en Ciudad Bolívar; ella cuenta con una gran sensibilidad social y, por tanto, su aporte en este proyecto fue vital. 
Frente a la dulzura del jazmín o del suave nardo

Son las manos quienes entre espinas las acercan

Y con delicadeza al olfato muy cerquita las procuran

Para sumergimos en tan delicioso bálsamo.

Al final de mis afanes, cuando mis fuerzas declinen

Y el corazón de una oración anheloso

Quisiera que tus manos sostuvieran mis manos

Y entrelazadas en mi pecho, las entregue al Poderoso.

(Acero, 2014)

Este primer apartado nos invita a repensar y de alguna manera a aprovechar los textos de la corporeidad. Al hacer una exégesis de los relatos de las mujeres, en la categoría cuerpo, descubrimos en la dimensión simbólica de las manos un nuevo horizonte de comprensión. La antropóloga Veena Das parece indicarnos la limitación del lenguaje cuando se apertura a uno nuevo:

Y si el lenguaje para la inefabilidad del dolor siempre se queda corto ante mi necesidad de su plenitud, entonces ¿̇es esta la sensación de desencanto que tienen los seres humanos con ellos mismos y con el lenguaje que les es dado? También, ¿implica la tarea de convertirse en un ser humano, incluso de convertirse en perversamente humano, una respuesta (aun si es la rabia) al sentido de pérdida cuando el lenguaje parece fallar?

(Veena, 2008, p. 348)

\subsection{Aprendizajes de la corporeidad como texto teológico. Hacia una TEOLOGÍA SIMBÓLICA}

Es importante recordar el contexto donde nacen estas expresiones, después de un largo proceso de conocimiento y compromiso con la población.

Ubicadas en el comedor de la Cámara de Comercio, la Facultad de Teología, a través del programa Vidas Móviles (Becerra, 2015, pp. 25-33), que tiene como objetivo acompañar, orientar y atender, compasiva e integralmente, a las personas y familias en situación de desplazamiento forzado, propuso un taller de Cuerpo y Sanación. Al principio tuvimos dificultades para trabajar con un grupo constante. Con el tiempo comencé a entender el nombre de ese programa pues es una población demasiado fluctuante, a veces sí y a veces no. Hay entusiasmo y compromiso durante una semana, a la siguiente, no aparece nadie; además, en este manejo del tiempo particular casi propio deben acudir a llamados de otros programas, talleres de estimulación, entrega de mercados, citas médicas que pueden demorar todo un día, en fin, miles de variables que son parte de la dinámica de la vida en Ciudad Bolívar. Finalmente se logró conformar un grupo de 20 mujeres, cada una con su propia historia, de diferentes edades, pero todas madres; 
algunas, abuelas de varios nietos. De variada edad y procedencia, las unía el deseo de aprender "algo nuevo", siempre pensando en ayudar a su familia; es increíble la petición, casi a gritos, de «ocuparse en algo». Una de ellas comentaba: «después de hacer el oficio de la casa me queda mucho tiempo, me deprime no tener nada qué hacer, yo estoy hecha para el trabajo, desde pequeña trabajaba la tierra, siempre tenía oficio, además quisiera aportar dinero, puesto hay muchas necesidades». Decidimos, entonces, vincular el taller de Cuerpo y Sanación con el aprendizaje de las manualidades.

Las mujeres en los encuentros y en los talleres, en tres diferentes momentos $(2009,2013,2015)$ comenzaron a valorar sus manos, de hecho, se conformó un grupo para tejer proyectos en común.

Durante los encuentros y a través de sus manos, poco a poco fueron valorando lo que hacían con papel reciclado y material desechable, lo que se convirtió en un símbolo de reconstrucción personal. Lo que eran desechos, aparentemente, lo convirtieron en obras de arte; así con acompañamiento espiritual y escucha personal, muchas mujeres fueron valorando sus vidas y valorando también la fortaleza del trabajo comunitario.

Ellas reconocen sus debilidades, reconocen que sus manos han maltratado y han hecho daño, pero también las reconocen como posibilidad de caricia, oración y reconstrucción comunitaria. A través de las manos muchas de ellas comenzaron a valorase, incluso a arreglarse y a centrarse en el autocuidado y cuidado de sus compañeras, lo cual representó un inicio de una dinámica comunitaria.

Si pudiéramos hacer una exégesis de las expresiones de estas mujeres, encontraríamos que su ejercicio manual, el acompañamiento espiritual y el trabajo comunitario, las llevó a reconocer su originalidad, su capacidad creativa y su sentido de responsabilidad, de lucha y de resiliencia. También son fuente de solidaridad, de cuidado; en términos de fe, de oración, de contemplación y alabanza.

Este punto en especial nos lleva a indagar otra manera de hacer teología. En este sentido encontramos autores como Spidlik y Rupnik, una luz para estudiar e investigar.

En su libro El conocimiento integral, la vía del símbolo estos autores, a partir de la obra artística, comienzan a dar validez a una teología mucha más integradora:

El pesado Dionisio Areopagita, en dos de sus obras, principalmente: Sobre los nombres de Dios y Sobre la teología, sabe perfectamente que, para conocer a Dios en sentido cristiano, no es suficiente con proponer las vías racionales que demuestran el Primer Motor del universo, la Primera Causa de todos los acontecimientos. El verdadero teólogo trata primero de recoger los predicados que la Biblia atribuye a Dios- por ejemplo, luz, vida, fuerza, bondad, belleza. —...No obstante, descubre 
muy pronto que ninguno de estos nombres expresa plenamente el gran misterio de Dios. La postura que expresa esta experiencia es la teología apofática que no teme profesar su propia ignorancia. Aun así, ésta ignorancia sagrada no es oprimente, no desanima, sino que, por el contrario, nos introduce en el arte de comprender los términos revelados como parábolas narradas por el Señor, que nos empujan a buscar un sentido más profundo de lo que aparece superficialmente.

(Spidlik \& Rupnik, 2010, pp. 41-42)

\section{EJEMPLIFLICACIÓN DE UNA TEOLOGÍA SIMBÓLICA A TRA- VÉS DE LAS MANOS Y EL TOCAR}

El tacto es el auténtico punto de encuentro entre los sujetos. Al igual que sucede en la vida cotidiana, en la que se extiende una gama de vivencias que van desde la violencia hasta la ternura, también la experiencia táctil puede abarcar desde el agarre y la aprehensión hasta el roce y la caricia. Dicotomía en la que a diario nos debatimos. La mano, órgano humano por excelencia, sirve tanto para acariciar como para agarrar. Mano que agarra y mano que acaricia, son dos facetas extremas de las posibilidades de encuentro interhumano... Cuando agarro un objeto lo hago sin pedir consentimiento, suponiendo que las cosas deben estar dispuestas a mi servicio en el momento en que las requiero.

(Restrepo, 1994, p. 54)

\subsection{LAS MANOS EN LA BIBLIA COMO SIGNO DE MISERICORDIA Y COMPASIÓN}

En el libro del Génesis 2,7 se señala que: «Dios formó al hombre con polvo del suelo e insufló en sus narices aliento de vida y resultó el hombre un ser viviente». Este relato permite deducir que Dios fabricó todo a través de sus $\operatorname{manos}^{20}$, ya que con ellas él forma, moldea y toca al hombre ${ }^{21}$. Se podría

20 El hermoso mito de la creación presenta a Dios como alfarero amoroso moldeando al ser humano y a todo ser viviente con tus propias manos. Bellísima imagen de un amor que se hace manos que acarician el barro y lo convierten en imagen y semejanza de su creador, en una realidad buena en sí misma: «Entonces el Señor Dios modeló al ser humano de arcilla del suelo... modeló de arcilla todas las fieras salvajes y todos los pájaros del cielo...» (Gn 2,7.19); "Y vio Dios que era bueno». Y dijo Dios «Hagamos al ser humano a nuestra imagen y semejanza... a imagen de Dios los creó; varón y hembra los creo» (Gn 1,25b-27). Bella imagen que te muestra como Partera de Vida (Martínez, 2006, pp. 45-92).

21 El A.T no tiene miedo de aplicar el lenguaje antropomórfico incluso a Yahvé. No es raro que la Biblia hable de la «boca» de Dios, de su "mirada», de sus "oídos», (ex 3,3-14), de su «brazo» que salva o de su «mano». la diferencia del Dios de la Biblia y de los dioses paganos está precisamente aquí: "Yahvé es sensible a los 
establecer que el ser humano «es una creatura que sale de las palabras y las manos de Dios» (Barrios, 2011, p. 40).

«Al igual que el lenguaje o la música, la mano nos habla...es uno de los medios más expresivos con que cuenta el lenguaje humano...» (Aubin, 2013, p. 104). La evolución no está dada sólo por la fabricación de herramientas, tal como nos indicaba el apartado anterior, sino por la contención y la caricia. Es decir, evolucionamos por la posibilidad de tocarnos, contenernos y cuidarnos...

«La mano se tiende hacia el cuerpo del otro, tratando de conjugar la distancia y abolir la separación, adaptando sus formas a la caricia y el masaje» (Aubin, 2013, p. 104) ... Ninguno de los órganos del cuerpo se puede sentir con tanta fuerza, el calor, el frío, el dolor o el placer...por la piel sentimos sin más que estamos vivos.

Nuestras manos son el mejor vehículo que tenemos para relacionarnos con el mundo pues extendidas nos permiten ensanchar el espacio que nos rodea. Llevamos inscrita en nuestras manos la opción fundamental que ha adoptado nuestra vida, ellas revelan nuestras inquietudes, nuestra generosidad. Son imagen externa de nuestro corazón endurecido o sensible.

(Gómez, 1997, p. 252)

"A mi vuelta, si los cuidados han exigido una suma mayor, te lo pagaré» (Lc 10, 35), dice el buen samaritano al posadero. Es un compromiso que va más allá y que no termina en el tiempo (Gómez, 1997, p. 53) o como el poeta José Benjamín lo expresa:

Ponme tus manos en los ojos
Para guiarme como un ciego
Por el fantasmal laberinto
De mi oscuridad y silencio.

(Gómez, 1997, p. 52)

Las manos, en los relatos del Evangelio, se convierten en protagonistas:

i. Cuando lava los pies a sus discípulos, Jesús los toma en sus manos y de alguna manera entra en un conocimiento íntimo de su ser (Jn 13, 1-20) (Gómez, 1997, p. 52).

ii. En la parábola del samaritano se hace concreta su compasión cuando cura las llagas del caído. Cuando le tiende la mano (Lc 10, 25-37).

iii. Tanto a la suegra de Pedro como a la hija de Jairo la toma de la mano y las levanta de su enfermedad (Mc 5, 21-43).

acontecimientos humanos, interviene, se compromete con la historia de Israel en un movimiento descendente» (De Candia, citado en Buitrago, 2015, p. 19). 
iv. La hemorroísa extiende su mano para tocar el manto de Jesús y sanarse (Lc 8, 43 -48).

La mujer del perfume rompe con sus manos el frasco de perfume y unge los pies de Jesús como símbolo de adoración y reconocimiento en una intimidad máxima (Mc 14,3-9).

v. María en el sepulcro estira su mano ante el resucitado para tocarle y regresarlo de nuevo (Jn 20, 1-18) ...como prolongación de la amante del Cantar de los Cantares "encontré luego al amado de mi alma, me agarré a él y no lo dejaré irse» (Ct 3,4).

vi. Tomás busca certezas a través del contacto (Jn 20, 19 -31).

vii.Para San Buenaventura... Dios entra a nosotros a través de los sentidos, «El hombre que recobra sus sentidos espirituales abraza la soberana suavidad bajo el aspecto del verbo encarnado que habita en nosotros corporalmente y, en su ardiente caridad, se deja tocar, besar y abrazar por nosotros» (Aubin, 2013, p. 121).

La característica del tocar de Dios es la Misericordia... no es un toque cualquiera... tiene la particularidad de trascender cualquier circunstancia... En tiempo de Jesús los leprosos simbolizaban a los intocables por su aspecto y mal olor... y aun así ante la súplica, el Señor extendió su mano y los tocó... «la Madre Teresa vivía de un modo muy concreto ese contacto de Jesús. Ella solía decir: cuando toco los miembros fétidos de los leprosos, sé que estoy tocando el cuerpo de Cristo.»(Aubin, 2013, p. 120).

Es decir, que allí hay un doble movimiento: si Jesús nos muestra la caricia de Dios, en términos de misericordia, con la caridad tocamos a Dios mismo... "Porque tuve hambre, y me disteis de comer; tuve sed, y me disteis de beber; fui forastero, y me recogisteis; estuve desnudo, y me cubristeis; enfermo, y me visitasteis; en la cárcel, y vinisteis a mí» (Mt 25,31) Nosotros cerramos el círculo amoroso con Dios cuando somos misericordiosos y caritativos.

En este camino de hacer de nuestro cuerpo un lugar de revelación de Ti, ocupan un papel especial las manos.

Las manos nos hacen artesanas de la cotidianeidad, transformando lo monótono, caduco y trivial en un lugar de creatividad y de amor. En este arte llevamos ventaja las mujeres. ¿Quién cómo nosotras sabemos de ello?: poner amor y ternura en la belleza que no dura, en la comida que desaparece, en la ropa que se arruga de nuevo, en el orden que se desordena, en la limpieza que no brilla nunca. Es 'un arte amoroso' que merece la pena aprender.

Dar vida, a través de nuestras manos, tiene que ver con algo que siempre hemos hecho las mujeres y que cada vez cultivamos más: amasar en la cotidianeidad el pan de la sororidad; unirnos a otras manos para tejer el manto de la solidaridad; dar, compartir, no acaparar para sí; saber pedir conscientes de nuestra 
propia pobreza; acariciar con ternura y pasión; aguantar y sostener, también aprender a sostenernos a nosotras mismas, levantar al caído, curar heridas ungiéndolas con el ungüento de la entrañable ternura... Manos, al fin, que sin ruido y sin alarde, pasan por la vida echando una mano, «haciendo el bien» como se nos dice de Jesús ${ }^{22}$.

Las manos como símbolo de memoria, pero también de renacimiento, nos conducen a otro tema de igual importancia...construir de nuevo la realidad en medio del dolor...las manos procesan los duelos. Hemos descubierto, a lo largo de tres investigaciones, que las víctimas del desplazamiento tienen una gran necesidad de vivir los duelos, pero, sobre todo, estas mujeres que han perdido tierra, parientes, hijos y dignidad.

Todos los ritos antropológicos realizados sobre los ritos de que están en torno a la muerte de un ser humano coinciden en la importancia que las mujeres han tenido en ellos, a lo largo del tiempo y en los más diversos lugares. Probablemente este hecho proviene de que su familiaridad con otros momentos críticos de la vida como lo son el parto y la enfermedad les ha hecho expertas en situaciones liminales, en torno a las cuales han desarrollado sus saberes: partes, sanadoras, plañideras...Su situación repetida de impureza, debida a la menstruación y al parto, también fue la causa de que se las considera más adecuadas para tratar con la impureza del cadáver. Y así desde la antigüedad han sido las mujeres de la familia las encargadas de preparar al cadáver (lavar, vestir, ungir...) para la posesión hasta la sepultura que dirigían los varones. El papel de las mujeres en estos

${ }^{22}$ «Me gusta descubrir que algunas veces, los escritores bíblicos, a pesar de sus prejuicios patriarcales, han sido capaces de dejar aflorar la capacidad de nuestras manos de mujer de revelar algo de ti. Son textos que necesitan ser desescombrados para que puedan hablar con nitidez, pero a pesar de todo están ahí. Y además son presentadas como mujeres que actúan transgrediendo los mandatos y las normas sociales y religiosas imperantes. En uno de esos textos, perdido en medio del Éxodo, ese libro dominado todo él por la figura de Moisés, se nos narra la historia de Sifrá y Pua (Ex 1,15-22), dos parteras egipcias que hacen de sus manos un lugar para la defensa de la vida, transgrediendo sagazmente las órdenes de la máxima autoridad políticoreligiosa de su tiempo para salvar la vida de los niños hebreos. Ellas nos enseñan a hacer de nuestras manos un lugar para la vida, aunque tengamos que arriesgar a decir "no" a todos los faraones de turno. En los sinópticos se nos narra la historia de otra mujer anónima (Mc 5,21-43; Mt 9,18-26; Lc 8,40-56) que también tiene la osadía de transgredir las normas sociales y religiosas de su tiempo. Se la conoce con el nombre de la hemorroisa, la mujer con la regla permanente, que arriesgó a tocar con sus manos lo prohibido, y eso prohibido era nada menos que tocar al Maestro. Ella, la impura, podía manchar todo lo que tocase, y fue esa trasgresión valiente la que le devuelve la salud. Que esta mujer osada nos enseñe a no tener miedo a tocar la vida, la realidad, los cuerpos... rompiendo tabúes milenarios que sólo conducen a la muerte y son mantenidos por el miedo». (Martínez, 2006, pp. 45-92). 
ritos puede verse ya en las antiguas pinturas griegas; ha sido habitual, hasta hace bien poco, en nuestros pueblos.

(Bernabé, 2009, pp. 516-517)

Si algo hemos aprendido en estos procesos investigativos es la necesidad acompañar procesos de duelo de nuestras mujeres y tal vez también los nuestros... El hecho de elaborar cosas con las manos y de simbolizar en ello su propia restauración, fue el primer paso para superar los duelos. Pero otro aspecto igualmente importante es vivir los duelos en comunidad. Tejer juntas proyectos comunes y al mismo tiempo escucharse y llorar para ser contenidas resultó ser un espacio sanador para la persona y para el grupo.

Se descubre en esta dinámica:

«Una intención expresa de recordar, de rememorar la vida de quien ha muerto. Se hace memoria del muerto y de su vida, se re-memora ${ }^{23}$, es decir, se mira su vida desde un marco de comprensión más total y se le re-integra, en su nueva realidad, a la totalidad del grupo.

(Bernabé, 2009, p. 519)

Las manos, en estas dinámicas, reconstruyeron el sentido personal y comunitario.

Hemos comprendido entonces que la salvación se concreta en términos de contención. En palabras de Martínez: «que Tus Manos se hagan cuerpo en nuestras manos... Para hacerlas tejedoras, artesanas del Reino en la cotidianeidad» (Martínez, 2015).

A través de lo cotidiano se explica el reino...levadura, banquete, sembrado, lámpara. No son asuntos extraordinarios los que nos acercan a Dios... tal vez por eso los pobres entienden más rápidamente de que se trata, tal vez por eso estas mujeres con un poco de levadura...elevaron su corazón y se hicieron más solidarias...

Tejemos tu Reino cuando, nuestras manos, ungidas por el Espíritu, son capaces de no acaparar y compartir, no asir para sí, sino sostener al otro, apoyar, colaborar, acariciar, abrazar, curar... Manos tendidas sin ruido y sin alarde en ese cotidiano pasar por la vida echando una mano, «haciendo el bien» como se nos dice de Jesús.

Nuestras manos por la acción de tu Espíritu, aliento de vida, pueden transformarse en instrumentos que cuidan la vida de nuestro planeta y protegen la

${ }_{23}$ El juego de palabras que une la idea de rememorar con la de volver a dar unidad de conjunto y hacer parte de un todo, se ve mejor en inglés: re-member. En castellano existe la palabra remembranza donde quizá se ve más claro la relación entre memoria y «ser miembro de» un todo. Por su parte, re-cordar recoge el matiz de volver a experimentar y a conocer de nuevo (Bernabé, 2014, p. 519). 
biodiversidad. Nos pueden hacer artesanas de una cultura de la sobriedad y se pueden unir a otras manos para tejer el manto de la solidaridad y de la paz. Tú, tejedora incansable del Reino, concédenos la gracia de comprender y acoger que nuestras manos colaboran contigo cuando son capaces de transformar lo monótono, caduco y trivial en lugar de la creatividad y el amor. Cuando transformamos la cotidianidad en obra de arte. Tejemos el Reino cuando nuestras manos saben: "pedir, acoger lo que la vida nos trae o nos quita; elegir y empeñar en ello nuestra libertad; y ofrecer/se y esperar respetuosamente para saber si el otro acoge el don. $¿$ Sabemos, podemos pedir, extender nuestra mano y mostrar nuestra indigencia y necesidad del otro?; ¿Hemos aprendido el arte de acoger y no de controlar? ¿Expresan nuestras manos la libertad de quien no se siente prisionero/a del consumo, del acaparar, del poder...? ¿Han recibido nuestras manos el don de saber ofrecer sin imponer, es decir, esperar respetuosamente para saber si el otro acoge el don? Así nuestras manos recibirán el don de saber ofrecer sin sentirse fuerte, "rico", encima, con derechos. Si no es así quizá nuestras manos sepan dar, pero aún no han aprendido a ofrecer respetuosamente.

(Martínez, 2015)

\section{CONCLUSIONES}

i. Un paso más se ha dado en esta investigación. Si en la primera fase resaltábamos el cuerpo como símbolo, en esta segunda fase descubrimos la importancia y el significado de la corporeidad. El cuerpo es el microcosmos que refleja la realidad social.

ii. Encontramos en lo estético una dimensión que se debe rescatar como un camino vital para la construcción de la comunidad.

iii. Recuperamos el gesto de las manos y el simbolismo del tocar en los textos bíblicos como camino para incorporarse a la comunidad y comprender la corporeidad.

iv. Recuperamos en la narrativa de las mujeres la importancia de la contención para encontrar en ello un proceso transformador y sanador.

v. Tejimos nuevas posibilidades junto con los teólogo/as de acompañamiento y construcción de comunidad.

vi. Recalcamos la importancia del manejo de los duelos como un proceso sanador personal y comunitario.

vii. Reconocemos la cotidianeidad como espacio de posibilidad para entretejer futuro y solidaridad.

viii. Descubrimos en la teología simbólica una línea para trabajar y profundizar en lo estético como herramienta sanadora, un camino que intuimos profundo y rico. Todo un horizonte por explorar. 
A manera de cierre, queremos retomar el testimonio maravilloso de la carta de Juan:

Lo que existía desde el principio:

Lo que vimos con nuestros ojos y contemplamos,

Lo que oímos a propósito del verbo (o palabra) de vida,

Y lo que nuestras manos tocaron (lit: palparon), de eso damos testimonio...y os lo anunciamos, para que seáis solidarios con nosotros; pero, además, esa solidaridad nuestra lo es con el padre y con su Hijo Jesús, el mesías. Os escribimos esto para que nuestra alegría llegue hasta el colmo (1Jn1, 1-4).

(Mourlon, 1993, p. 15)

\section{REFERENCIAS BIBLIOGRÁFICAS}

Aubin, C. (2013). Las ventanas del alma. España: Sal Terrae.

Acero, R. (2014). Las Manos. Poema inédito. Bogotá: Pontificia Universidad Javeriana.

Barrios González, C. (2011). Imposición de manos: ¿Magia o símbolo sacramental? Tesis de Licenciatura en Teología. Bogotá: Pontificia Universidad Javeriana.

Becerra, S. (2015). El arte de re-iniciar la vida. Bogotá: Editorial Javeriana.

Bernabé, C. (2009). Palabras de mujeres en el inicio del Kerigma. Salamanca: Separata de Ciencia Tomista, pp. 516-517.

- (2014). El cuerpo como territorio de Salvación. Traducción de Rodríguez, A. Facultad de Teología. Bogotá: Pontificia Universidad Javeriana.

Borrego, J. (2015). Estética en Zubiri. [Versión digital] Recuperado de: http://www.javier.org.es/archivos/enlaces/_private/trabajos/est\%E9tica_en_xavier_zubiri1.htm.

Buitrago, W. (2015). La corporeidad como lugar de salvación. Tesis de Teología. Bogotá: Pontificia Universidad Javeriana.

Cardoso, N. (2015). Pautas para una hermenéutica feminista de la liberación. [Versión digital] Recuperado de http://www.claiweb.org/ribla/ribla25/presentacion.html.

Del Valle, C. (2012). Belleza y Liturgia. España: Sal Terrae.

Derrida, J. (2011). El tocar: Jean-Luc Nancy. España: Amorrortu Editores.

Gómez-Acebo, I. (1997). Cinco mujeres oran con los Sentidos. España: Desclee de Brouwer.

Grün, A. (2010), La experiencia de Dios a través de los sentidos. España: Sal Terrae.

Gutiérrez, A. (2012). Hacia la recuperación y sanación corporal: elaboración de violencias basada en artes de acción/artes creativas. [Versión digital] Recuperado de http://bdigital.unal.edu.co/6701/1/angelabeatrizgutierrezcabrera.2012.pdf

Lizcano, H. (2014). Oh mano blanda. Bogotá: Gráficas Iberia.

López Quintas, A. (1991). Para comprender La experiencia estética y su poder formativo. Navarra: Editorial Verbo Divino.

López Villanueva, M. (2010). Orar con el cuerpo y recibir el cuerpo. España: Sal Terrae, pp. 409-414. 
Martínez, E. (2015). Orar desde las relaciones laborales. [Versión digital] Recuperado de http://www.emmamartinezocana11.blogspot.com/2012/07/orar-desde-lasrelaciones-laborales.html.

- (2012). Te llevo en mis entrañas dibujada. Madrid: Narcea Ediciones.

Maturana, H. (2015). Emociones y lenguaje. [Versión digital] Recuperado de: http:// es.slideshare.net/FelipeLealBravo/emociones-y-lenguaje-humberto-maturana-1989280.febrero 27.

Mourlon, P. (1993). El hombre en el lenguaje bíblico. Pamplona: Editorial Verbo Divino.

Pastor, F. (1995) Antropología Bíblica. Navarra: Verbo Divino.

Peña, L. (2015). La estética dimensión olvidada de la Eucaristía. [Versión digital] Recuperado de: http://es.wikiversity.org/wiki/Origen_de_la_est\%C3\%A9tica

Porcile, M. T. (1995) La mujer, espacio de salvación. Madrid: Anzos.

Restrepo, L. (1994) El Derecho a la Ternura. Santa Fe de Bogotá: Arango Editores.

Roccetta, C. (1994). Hacia una Teología de la corporeidad. Madrid: San Pablo.

Rodríguez, A., y Sierra, A. (2009). Proyecto Manos de Esperanza. Revista Fronteras. Facultad de Teología, Pontificia Universidad Javeriana: Bogotá.

Rodríguez, A., y Sierra, A. (2013). El cuerpo como narración del sujeto en situación de desplazamiento. En: Vivir la fe en la Ciudad Hoy. Eckholt, Margit \& Stefan. Conferencia Episcopal Alemana.

Spidlik, T., y Rupnik, M. (2010). El conocimiento integral. La vía del símbolo. Roma: Editorial BAC.

Spretnak, y Ress, M. (1994). Ute Seibert-Cuadra, Lene Sjorup, (Eds). Del cielo a la tierra. Una antología de teología feminista. Chile: Editorial Sello Azul.

Toko-Pa. (2016) https://toko-pa.com/2016/01/12/the-handmade-life/.

Veena, D. (2008). Sujetos del dolor, agentes de dignidad. [Versión digital] Recuperado de www.bdigital.unal.edu.co/8285/.

Vélez, C. (2013). Ciudad y mujer una apuesta evangelizadora. Revista Evangelización y Culturas urbanas. Bogotá: Concejo Episcopal Latinoamericano. Vol. XXXI, 155, pp. 453-471. 